
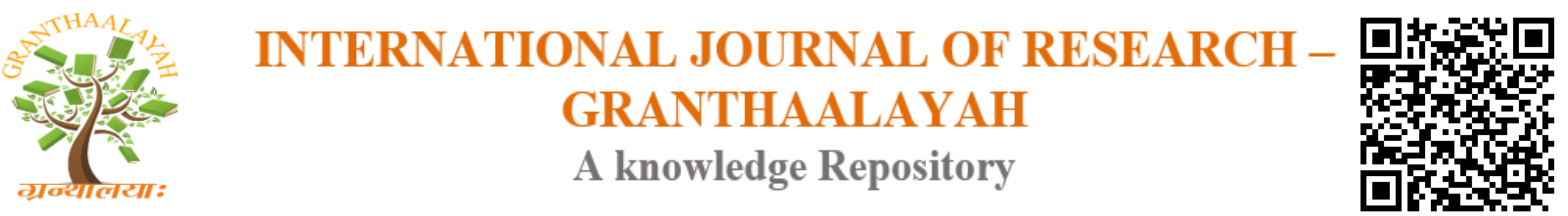

Science

\title{
CONCEPTUAL STUDY OF ROLE OF AYURVEDA IN PREVENTION OF LIFESTYLE DISEASES
}

\author{
Dr.Vijaylaxmi Ramprasad Reddy ${ }^{1}$, Dr.Ashwini A.Shitre ${ }^{2}$ \\ ${ }^{1}$ M.D (Scholar), Kaumarbhritya-Balroga Department, D.Y.Patil University, School of Ayurved, \\ Nerul, Navi Mumbai, India \\ ${ }^{2}$ M.S (Streerog and Prasutitantra), Professor \& H.O.D, Kaumarbhritya-Balroga Department, \\ D.Y.Patil University, School of Ayurved, Nerul, Navi Mumbai, India
}

\begin{abstract}
Ayurveda is the science of life, guiding in maintaining health and curing of diseases by balancing doshas-dhatus-malas and jatharagni. Ahara, nidra and brahmacharya form the pillars of health as per Ayurveda.

Lifestyle diseases are those diseases whose occurrence is based on the daily habits of people and are a result of an inappropriate relationship of people with their environment. The main factors contributing to lifestyle diseases include bad food habits, physical inactivity and disturbed biological clock. Occupational lifestyle diseases include those caused by the factors present in the vicinity of pollutants. These factors are also responsible for environmental allergies. So, a healthy lifestyle must be adopted with a proper balanced diet, physical activity and by giving due respect to biological clock.

Healthy lifestyle methods are now easily achievable with nutritional counselling, exercise training, de-addiction programmes, regular medical check-ups and stress management techniques. Ayurveda provides better solution in the guidelines of proper dietary management, lifestyle advises, panchakarma procedures like detoxification and bio-purification procedures, medicaments and rejuvenation therapies. In this revolutionized era, we cannot stop doing the developmental work, but we can certainly reduce our ailments with simple and effective measures in our lives as suggested in Ayurveda, the science of life.
\end{abstract}

Keywords: Ayurveda; Lifestyle Diseases.

Cite This Article: Dr.Vijaylaxmi Ramprasad Reddy, and Dr.Ashwini A.Shitre. (2018). "CONCEPTUAL STUDY OF ROLE OF AYURVEDA IN PREVENTION OF LIFESTYLE DISEASES." International Journal of Research - Granthaalayah, 6(3), 149-154. https://doi.org/10.29121/granthaalayah.v6.i3.2018.1508.

\section{Introduction}

Ayurveda is the science of life. It aims at maintaining health and curing diseases by maintaining balance in doshas-dhatus-malas and jatharagni ${ }^{(1)}$. A healthy diet contains fruits, vegetables, 
whole wheat products and a low content of saturated fatty acids that are required to meet the daily requirement of calories and for disease-prevention ${ }^{(2)}$

Disease is a state of discomfort in which the normal functioning of the body is affected (3) Lifestyle diseases is defined as the class of diseases caused partly by unhealthy behaviours and partly by other factors. A lifestyle is the pattern of living that we follow - how we work, what and when we eat, how and when we sleep, how much physical activity we do and whether we smoke or consume alcohol. . The incidence of lifestyle diseases like Hypertension, Diabetes Mellitus, Obesity and Cardiovascular Diseases is high on the rise.

The lifestyle factors associated with these diseases is of two types ${ }^{(4)}$

1) Modifiable (those that can be changed) like food habits, physical activity level, addiction (smoking, drinking) and stress.

2) Non-modifiable (those that cannot be changed) like age, gender and heredity.

Lifestyle diseases lead to reduction in productivity and development of the country. Therefore, there is an increasing concern for their prevention. Ayurveda is the life science with ways to prevent and manage lifestyle diseases. It provides better solution in the forms of proper dietary management, lifestyle advises, panchakarma procedures like detoxification and bio-purification procedures, medicaments and rejuvenation therapies.

Lifestyle of a person is a cumulative product of his physical capacity co-ordinated with psychological functioning, displayed in the form of habits, behaviour, dietary and living pattern. Thus, it involves a pure psychological and innate control over the physical and sensory activities. When the initiation, control and co-ordination are disturbed, it leads to the derangement of lifestyle and results in any of the lifestyle diseases. Ayurveda narrated this phenomenon as 'Prajnaparadha' ${ }^{(5)}$ There are ample improper actions as an impact of Prajnaparadha which are root causes of various diseases, e.g. , the habit of suppression of natural urge in improper lifestyle can be considered as one of the root causes of occurrence of lifestyle diseases. Removal of these accumulated waste products is the first line of treatment as described in Ayurveda. ${ }^{(6)}$

In the management of lifestyle diseases, Ayurveda offers various regimens including Dinacharya (daily regimen), Ritucharya (seasonal regimen), Panchakarma (five detoxification and biopurification therapies), and Rasayana (rejuvenation) therapies. The Sadvritta (ideal routines) and Aachara Rasayana (code of conduct) are utmost important to maintain a healthy and happy psychological lifestyle. ${ }^{(7)}$

Uncontrolled urbanization, increased pollution, lack of control over regulation of food quality and contents of adulteration and improper dietary-living pattern disturbing the biological clock, all form the main reasons for occurrence of lifestyle diseases. ${ }^{(8)}$

\subsection{Cardiovascular Diseases}

Cardiovascular diseases, a class of diseases that involve the heart and the blood vessels (arteries, capillaries, and veins) cause majority of deaths worldwide. Raised blood pressure approximately causes 7.5 million deaths throughout the world. The normal systolic and diastolic blood pressure is 140 and $90 \mathrm{~mm} \mathrm{Hg}$. Abnormal blood pressure levels have been shown to be related to the possibility of stroke. When blood supply to part of our brain is interrupted or severely reduced, 
thus depriving brain tissue with oxygen and food supply then there occurs an attack of stroke. Coronary heart disease as a result of deposition of fats on the inner wall of the coronary arteries that supply blood to the heart muscle leading to clogged arteries also increase the chances of stroke.

\subsection{Chronic Obstructive Pulmonary Diseases (COPD)}

This disease is characterized by increasing breathlessness due to persistent airflow limitation caused by different combinations of small-airway diseases. It includes a spectrum of conditions which include Emphysema, Chronic Bronchitis, Asthma and some other forms of Bronchiectasis. COPD primarily affects the structural units of pulmonary system by disrupting the mechanical process of respiratory air-flow and diffusion to blood. This chronic condition thickens and disrupts the elasticity of pulmonary air sacs (alveoli), erosion of inter-alveolar septum and secretes excess of mucus that clog the air passage. COPD can be managed well by lifestyle modifications such as quitting smoking, avoiding lung irritants, regular exercise and supporting treatment medicines such as bronchodilators, etc.

\subsection{Diabetes Mellitus (DM)}

Depending on the pathophysiology, DM can be classified into two types, Type-1 Diabetes Mellitus and Type-2 Diabetes Mellitus. Compared to T1DM, T2DM are less familial and mostly influenced by the environmental factors, unhealthy life style and food habits. T1DM comprises only $5 \%$ of total DM and seen among children or young adults who are unable to produce hormone insulin (insulin dependent DM, IDDM) due to loss of the insulin producing beta-cells of the Islets of Langerhans in the pancreas. T2DM are mostly found among adults where cells fail to respond to insulin. Several life style changes are mostly associated with obesity which indirectly influence the onset of T2DM. ${ }^{(9)}$ Insulin is the main hormone that regulates the glucose uptake by the muscle, adipose tissues and liver from circulating blood. Therefore, lower insulin level or disrupted insulin receptors both can deregulate the uptake of insulin from blood and necessary intracellular signalling. Elevated amount of blood glucose Affect the efficiency of kidneys and other body organs like, eyes, brain tissues, cardiac tissue, distal parts of hands and legs. Common co-morbidities associated with DM are Hypertension, Dyslipidemia, Nonalcoholic Fatty Liver Disease, Cardiovascular Disease, Kidney Disease, Retinopathy, etc, which lowers the quality of life.

Symptoms of DM are associated with physical abnormalities such as, early fatigue, frequent urination, excessive thirst, weight loss, increased huger, lack of interest and concentration, slow healing wounds, etc. Generally symptoms are very prominent and sudden in case of T1DM in comparison to T2DM. Diagnosis of DM is mostly done by testing the circulating glucose level in the blood in fasting condition or after 2 hours post-meals. FPG level between 70-99 mg/dl are categorised as normal, $>125 \mathrm{mg} / \mathrm{dl}$ are diabetic and individuals in between these threshold are grouped as pre-diabetic individuals who has higher risk of developing DM over the time. Similarly for PP glucose level threshold for DM is $>140 \mathrm{mg} / \mathrm{dl}$. Other commonly performed tests are haemoglobin A1c (HbA1c) and oral glucose tolerance test (OGTT). Random plasma glucose (RPG) test, is sometimes performed to diagnose Diabetes Mellitus in regular health check-up camps. Controlling blood pressure and serum lipid levels and reduced stress conditions are also 
important measures towards preventing the Diabetes Mellitus onset. Commonly used first line of drugs includes Metformin, aspirin, angiotensin converting enzyme inhibitors (ACEIs) etc. Late stage T2DM and T1DM is typically treated with the Insulin injections.

\subsection{Alzheimer's disease (AD)}

$\mathrm{AD}$ is characterized by irreversible and progressive loss of memory and other intellectual abilities that can affect daily life. Commonly found in older people (age $>60 \mathrm{yrs}$.). Dr. Alois Alzheimer first noticed changes in the brain tissue of a woman who died due to an uncommon mental disease. Formation of extracellular amyloid plaques and intracellular neurofibrillary tangles are the primary features of $\mathrm{AD}$ which causes the significant shrinkage of brain size and loss of connection between nerve cells in the brain.

Based on the pattern of memory loss and degree of neuronal impairment of the brain AD can be categorized into four stages namely (i) Pre-dementia, (ii) Early AD, (iii) Moderate AD. Blood testing and brain imaging are done to detect AD. Sophisticated brain image scanning like CT (computed tomography) and MRI (Magnetic resonance imaging), SPECT (Single photon emission computed tomography) and $\mathrm{PiB}$ (Pittsburgh compound $\mathrm{B}$, a radioactive compound) mediated PET (positron emission tomography) are necessary to detect the brain tissue damage and formation of amyloid plaques.

Several drugs are available which can be used to slow-down the progress of AD. Early AD can be treated with Donepezil and Bapineuzumab. Modified life style including regular meditation, change in diet and physical activities prove beneficial in delaying the onset and progress of the disease.

\subsection{Cancer}

The WHO reported 12.7 million new cancer cases reported in the year 2008 and forecasted 21.4 million new cases by 2030, with nearly two thirds of all cancer incidences reported from lowand middle-income countries. In upper middle-income and high-income countries, prostate and breast cancers are the most frequently diagnosed in males and females respectively, while lung and colorectal cancers is the second most common cancer respectively. Lung cancer is the most common cause in both sexes. In low-income countries, the lung and breast cancers remain the most commonly diagnosed cancer.

Insufficient physical activities, unhealthy eating habits and stress has also been associated with this life-threatening disorder. ${ }^{(10)}$ Researchers have found that there is a direct association among higher levels of alcohol consumption and increasing risk of few cancers, liver diseases and cardiovascular diseases. Major cancers can be prevented by improved balanced diet, proper physical activities and healthy practices.

\section{Discussion}

The advancement of our lifestyle with dependency on technology and gadgets directly have an impact on our health. Heavy computer use, suspected glaucoma and short-sightedness appear to 
be interlinked. Regular spending of lot of time in front of computer may lead to neck and back pain. The stiffening of neck also is a common problem along with headache, fatigue and exhaustion. Wrong sitting or standing posture while working gives strain to the backbone and gives a chronic back pain. Other extensively used gadget is mobile phone. Research conducted on animals in different countries has established a number of adverse effects of mobile phone radiation like low fertility, reduction in attention and memory, sleep disturbances, headache, ringing in the ears, hearing disturbances etc.

People working in night shifts and those indulging in late night parties suffer from a disturbed biological clock leading to insomnia, indigestion, acidity, loss of appetite, headache, irritability, hypertension, mood fluctuations and body pain.Bad public transportation and faulty office postures for quite a long time can lead to a chronic back pain and spondylolysis. People working in high temperatures face problems related to BP, metabolism and organ failure due to shock. Extreme cold working condition causes hypothermia and shock. Children who grow up to be obese have a high risk of development of early heart diseases, and hypertension.

Observations in the new lifestyle are: ${ }^{(11)}$

1) Majority of young population involved smoking and drinking.

2) Fresh fruits and vegetables substituted by junk foods.

3) Use of bus or car instead of walking even for short distances.

Some healthy tips are:

1) Use stair-case instead of lift or escalator.

2) Take a balanced diet at proper meal times.

3) Avoid processed and packaged foods.

4) Drink sufficient amount of water.

5) Practice yoga or meditation to avoid stress in life.

6) Keep away from smoking and drinking alcohol.

Certainly the urbanization process has caused poor lifestyle choices, such as smoking, overuse of alcohol, poor healthy diet, insufficient physical activity and chronic stress, leading to development and progression of various lifestyle diseases. ${ }^{(12)}$ Healthful habits help prevent many-of-these lifestyle diseases (chronic medical disorders). Healthy lifestyle methods are easily achievable with appropriate interventions, like nutritional counselling, exercise training, deaddiction programmes, regular medical check-ups and stress management techniques.

\section{Conclusion}

A healthy lifestyle must be adopted to combat these diseases with a proper balanced diet, physical activity and by giving due respect to biological clock. To decrease the ailments caused by occupational postures, one should avoid long sitting hours and should take frequent breaks for stretching or for other works involving physical movements. In this revolutionized era we cannot stop doing the developmental work, but we can certainly reduce our ailments by incorporating these simple and effective measures to our lives. Hence Ayurveda has a major role to play in prevention of lifestyle diseases. 


\section{References}

[1] Vagbhata Ashtanga Hridayam (Text with English Translation, Notes, Appendices and Index) Editor and Translator Prof.K.R.Srikantha Murthy, pages 3-8.

[2] Sobra J, Ceska R. Diseases of civilization from the aspect of evolution of the human diet.

[3] Sushruta Samhita (Text,with English Translation, Notes, Appendices and Index)Vol I Translater Prof. K. R.Srikantha Murthy, ChaukhambhaOrientalia, Varanasi, Re-print,m2012. 15:38

[4] Choi, Bernard C.K., Hunter, David J., Tsou, Walter and Sainsburgy,Peter (2005), "Diseases of comfort: primary causes of death in the22nd century", Journal of Epidemiology and Community Health,Vol. 59, No.12, pp.1030-1034.

[5] Charaka, Charaka Samhita (text with English translation) Editor and Translator P.V. Sharma, Chaukahamba Surabharati Prakashan, Varanasi, Re-print, 2010.p. 10

[6] Vagbhata Ashtanga Hridayam(Text with english Translation) Editor and Translator Prof.K.R.Srikantha Murthy, p.225-265

[7] Vagbhata Ashtanga Hridayam(Text with english Translation)Editor and Translator Prof.K.R.Srikantha Murthy, p.3-52

[8] Preventing non communicable diseases in the workplace through diet and physical activity:WHO/World Economic Forum report of a joint event. 2008. ISBN 978924159632

[9] Anoop Misra, Lokesh Khurana. Metabolic Syndrome and Related Disorders. ahead of print. doi:10.1089/met.2009.0024.

[10] Lichtenstein P, Holm NV, Verkasalo PK. Environmental and heritable factors in the causation of cancer.

[11] Deepika Daily (2012), "Rogangal Kshanichuvaruthan fast food kazhikkuka", April 2, p.6.

[12] ASSOCHAM Study on Preventive Healthcare. 2009

*Corresponding author.

E-mail address: reddyvijaylaxmi3@gmail.com/dr.ashwini68@gmail.com 